\title{
Cross-Validation of the Foundation Pain Index with PROMIS-29 in Chronic Pain Patients
}

\author{
Jason E Pope' \\ Michael A Fishman (D) ${ }^{2}$ \\ Joshua A Gunn ${ }^{3}$ \\ Bradley $M$ Cotten (D) $^{3}$ \\ Melissa $M$ Hill (iD ${ }^{3}$ \\ Timothy R Deer (D) ${ }^{4}$ \\ 'Evolve Restorative Center, Santa Rosa, \\ CA, USA; ${ }^{2}$ Center for Interventional Pain \\ and Spine, Lancaster, PA, USA; ${ }^{3}$ Ethos \\ Research and Development, LLC, \\ Newport, KY, USA; ${ }^{4}$ The Spine and \\ Nerve Center of the Virginias, \\ Charleston, WV, USA
}

Correspondence: Jason E Pope

Evolve Restorative Center, Santa Rosa, CA, USA

Tel + I844 527-7369

Email jepope@evolverestorativecenter. care
Purpose: Discovery and validation of pragmatic biomarkers represent significant advancements in the field of pain management. Evaluating relationships between objective biomarkers and patient-reported outcomes (PROs) is an effective way to gain mechanistic insight into the potential role of biochemistry in chronic pain. The aim of this study was to validate the Foundation Pain Index (FPI) by evaluating associations between deranged biochemical function and PROMIS-29 domains in individuals living with chronic pain.

Patients and Methods: PROMIS-29 scores and FPI test results were obtained from 298 patients with chronic pain in this retrospective, observational study. Statistical analysis was performed using clinical test data to evaluate relationships between deranged biochemical function and quality of life measures across 8 universal domains.

Results: FPI scores significantly associated with multiple PROMIS-29 domains including physical function, impact score, fatigue, pain interference, and depression $(\mathrm{P}<0.05)$. Moreover, specific analytes that comprise the FPI significantly correlated with PROMIS-29 domains, including 5-hydroxyindolacetic acid (pain interference, physical function, and pain impact scores), hydroxymethylglutarate (physical function), homocysteine (pain impact scores), kynurenic acid (pain interference and physical function), and quinolinic acid (physical function) $(\mathrm{P}<0.05)$.

Conclusion: Cross-validation of the FPI with PROMIS-29 domains further supports the role of deranged biochemical function in the etiology of chronic pain. Objective identification of atypical biochemical function and subsequent correction holds tremendous promise for the non-opioid management of pain. Continued research efforts will aim to determine the impact of biochemical optimization in pre-surgical periods and post-surgical outcomes in patients with chronic pain.

Keywords: biomarker, pain, oxidative stress, neurotransmitter, micronutrient deficiency, kynurenine pathway

\section{Plain Language Summary}

Validation of mechanistic biomarkers would improve the assessment, treatment, and management of chronic pain in patients. Evaluating the relationships between validated biomarkers and Patient-reported Outcomes (PROs), such as the PROMIS-29 instrument, could reveal underlying, biochemical conditions driving characteristics of chronic pain. The Foundation Pain Index (FPI), a multi-biomarker assay based on algorithmic analysis of abnormal urinary metabolites, was found to correlate with multiple PROMIS-29 domains, such as fatigue and depression. Specific components of the FPI associated with assessments of physical function, pain interference, and pain impact scores. Laboratory analysis of patient urine samples revealed the majority of subjects to have $\geq 2$ abnormal biomarker findings. This study supports the role of biochemistry coupled with clinical assessments of chronic pain to 
provide medical providers opportunity to apply targeted, nonopioid interventions to patients. Furthermore, these tests could provide clinicians a course-of-action to optimize perturbed biochemistry in pre-surgical periods and improve surgical outcomes in patients.

\section{Introduction}

Chronic pain is an overwhelming public health burden impacting $20-30 \%$ of Americans and representing total societal costs of $\$ 600$ billion a year. ${ }^{1,2}$ Diagnosis and treatment of chronic pain is complicated by the absence of a linear relationship between the type or degree of organic pathology and pain intensity. Instead, the individual experience of chronic pain is shaped by a myriad of biochemical, psychosocial, and behavioral factors. ${ }^{3}$ Given the biopsychosocial complexity of chronic pain, the reliance on patient self-report, and the risks associated with long-term opioid medications, it is imperative that comprehensive evaluation tools which optimize decisionmaking and minimize risk are available to all clinicians. Patient-reported outcome (PRO) measures are instruments for documenting patients' perceptions of disease and treatment impact on their health and function. In the context of pain management, PROs can be employed to track progress, evaluate treatment efficacy, and change the course of care when necessary. Traditionally employed in a clinical trial setting, these multidimensional assessment tools are now finding widespread utility in clinical practice for the evaluation of chronic pain patients. In an active clinic setting, PRO instruments are available to assess several aspects of pain management, including overall symptom burden, mental health (eg depression, anxiety, stress, personality, and positive and negative affect), physical function, mental function, disability, and social satisfaction and impairment. ${ }^{4,5}$

Pain management, a specialty often dependent on flawed measurements such as linear numerical rating scales (NRS) and visual analog scales (VAS), is now part of a growing patient-centric movement that places majority of focus on multidimensional patient reported outcomes. This strategy of assessing pain in the context of biopsychosocial assessment is recommended by the Health and Human Services (HHS), ${ }^{6}$ National Institutes of Health (NIH), ${ }^{7}$ Institute of Medicine, ${ }^{8}$ Initiative on Methods, Measurement, and Pain Assessment in Clinical Trials (IMMPACT), ${ }^{9-11}$ and others.

Our group recently reported on the clinical validation of a multi-biomarker assay for the evaluation of chronic pain in patients. ${ }^{12}$ This innovative laboratory test, known as the Foundation Pain Index (FPI), evaluates eleven endogenous biomarkers, which represent a highly diverse set of biochemical pathways involved in the pathogenesis of chronic pain. ${ }^{12}$ Quantitative results for each of the eleven biomarkers are analyzed via a proprietary algorithm to generate a single numerical score (FPI score) on a scale of 0-100, which describes the severity of abnormal findings. FPI scores provide clinicians with objective and reproducible measures of the biochemical, metabolic, and nutritional drivers of chronic pain. Criterion validity of this multi-biomarker pain index was established by demonstrating a significant correlation between FPI scores and a validated PRO instrument known as the 36-item short-form health survey (SF-36). The SF-36 survey is a validated assessment of chronic pain that measures health-related quality of life across eight dimensions (physical functioning, physical role, bodily pain, general health, vitality, social functioning, emotional role, and mental health). Specifically, our group was able to show that deranged biochemical function, evident by higher FPI scores, was significantly correlated to overall SF-36 scores $(\mathrm{P}=0.0141)$, general health $(\mathrm{P}=0.0457)$ and even more significantly, with emotional well-being $(\mathrm{P}=0.0044)$ and limitations due to emotional problems $(\mathrm{P}=0.0011) .{ }^{12}$ To our knowledge, this is the first report of a validated multibiomarker test panel that has been shown to correlate with PRO measures. These findings provide strong evidence for a role of perturbed biochemical function in the etiology and/or chronicity of pain. During initial validation of the FPI, the SF-36 was selected as the most appropriate validated assessment to evaluate both the physical and emotional impact of pain on subjects. Since publishing this data, our group has received requests from practicing physicians to cross-validate the FPI with PROs more commonly used in the clinic setting.

A common measurement language is critical to galvanizing innovation, research, and care improvement. The Patient Reported Outcomes Measurement Information System (PROMIS) was created by the NIH in 2004, resulting in the initiation of several studies that focused on heart failure, chronic obstructive pulmonary disease, rheumatoid arthritis, cancer, chronic low back pain, and major depression. The goal of PROMIS was to update longer form legacy instruments with validated outcomes measures that provided greater accuracy from a reduced questionnaire. Many familiar legacy measures were used to create the PROMIS item banks, and crosswalk tables 
are available to allow for comparisons (PROsetta Stone ${ }^{\circledR}$; http://www.prosettastone.org). The PROMIS-29 battery assesses mental health, physical health, and social health through seven 4-question instruments, including assessments of fatigue, pain intensity, pain interference, physical function, sleep disturbance, anxiety, depression, and ability to participate in social roles and responsibilities. The PROMIS-29 and its subdomains have been validated and/or cross-walked to a host of legacy measures in a variety of populations, including the Oswestry Disability Index, Roland-Morris Disability Questionnaire, Short form-36, Brief Pain Inventory-Pain Interference domain, the EQ-5D, and Quality-Adjusted Life Year calculations.

The present study sought to validate the FPI by evaluating relationships and associations between deranged biochemical function and PROMIS-29 domains. Previous work has highlighted strong correlations between PROMIS-29 and SF-36 scores, and as such we hypothesize that validation of FPI against a second PRO would further support the role of deranged biochemistry in the etiology of chronic pain. ${ }^{13,14}$

\section{Patients and Methods Study Subjects}

Patients were selected for this retrospective, observational study if they had completed a PROMIS-29 survey and provided a urine sample within 15 days of the initial encounter for pain biomarker testing before treatment initiation. Subjects were selected from a single clinical site in Santa Rosa, CA, and a total of 298 unique patients were included in the analysis. Subjects were excluded from the analysis if they were being prescribed medication(s) known to directly modulate any of the component biomarkers of the Foundation Pain Index assay or if they had a history of hepatic and/or renal disease. For this study, no patients were excluded based on medications or history of hepatic and/or renal disease. IRB exemption for the present study was approved by the Western Institutional Review Board (IRB Study Number: 1289960). Research was performed in compliance with the Declaration of Helsinki and respected the confidentiality and anonymity of patient health information.

Researchers referred to the International Association for the Study of Pain (IASP, 2020) for defining chronic pain as "An unpleasant sensory and emotional experience associated with actual or potential tissue damage, or described in terms of such damage."15 For this study, pain was identified as chronic if symptoms persisted for longer than 3 months. Despite presumed differences in diagnosis, duration of pain, and previous treatment of pain-related syndromes, the study subjects were assessed as a homogenous group.

\section{PROMIS-29}

The PROMIS-29 is a 29-item profile instrument that assesses 8 universal domains (not disease-specific): Physical Function, Anxiety, Depression, Fatigue, Sleep Disturbance, Ability to Participate in Social Roles and Activities, Pain Interference, and Pain Intensity. The first seven domains are assessed with 4 questions each; Pain Intensity is measured with a single 11-point numeric rating scale (NRS) from 0 (no pain) to 10 (worst imaginable pain). High scores represent more of the domain being measured. Thus, on symptom-oriented (negatively worded) domains of PROMIS-29 (anxiety, depression, fatigue, pain interference, and sleep disturbance), higher scores represent worse symptomatology. On the functionoriented (positively worded) domains (physical function and social role) higher scores represent better functioning.

To score the PROMIS-29 domains, item responses were tallied, multiplied by the total number of category questions, and divided by the number of patient responses. T-scores were then calculated for all domains except the single pain intensity item, which was used in raw form. The T-score converts the raw score into a standardized value, which can be directly compared to the general US population. For most PROMIS instruments, a score of 50 is the average for the general US population with a standard deviation (SD) of 10 . All study subjects completed a PROMIS-29 survey upon admission into the clinical site chronic pain management program and immediately before providing a urine sample for biomarker analysis.

\section{Pain Impact Score (PIS)}

The Pain Impact Score (PIS) is calculated from the PROMIS-29 pain intensity, physical function, and pain interference subdomains. The raw scores of these measures are combined to define a single digit score. The raw score for physical function is inverted so that higher raw scores denote worse function. The raw score ranges from 0 to 10 for pain intensity and 4 to 20 for pain interference and physical function. The PIS ranges from 8-No Impact (pain intensity of 0 , pain interference of 4 , physical function of 4) to 50-High Impact (pain intensity of 10 , pain interference of 20 , physical function of 20$)^{7}$ 


\section{Sample Collection}

Urine samples for FPI testing were collected within 15 days from patients on their initial clinic visit after completing the PROMIS-29 survey. After collection, urine samples were packaged and shipped to Ethos Laboratories (Newport, KY) for comprehensive biomarker analysis and FPI score derivation.

\section{Analytical Methodologies}

Samples were accessioned into a centralized laboratory database (LIS) and prepared for analysis. Each 96-well plate contained two quality controls, four-point calibrators, and one negative sample when measuring excreted metabolites in patient samples. Liquid chromatography tandem mass spectrometry (LC-MS/MS) assays were used to determine biomarker creatinine concentrations. All analytes were analyzed with MassHunter software and quantified using isotopic dilution. Biomarker concentrations are reported as $\mu \mathrm{g} / \mathrm{mg}$ creatinine, with normalization to creatine correcting urinary dilution.

\section{Statistical Methods}

Descriptive statistical analysis for the patient cohort was analyzed by GraphPad Prism (8.3.0). Mean, standard deviations, T-scores, and ranges (minimum to maximum values) were assessed among patients with chronic pain. Reference ranges calculated from a previous study were used. ${ }^{16}$ Due to non-normal distribution of PROMIS-29 assessments among patients with chronic pain, Spearman's rank coefficient (r) was used (GraphPad Prism 8.3.0). Correlation analysis was performed for group characteristics (age, sex, creatinine levels, biomarker concentrations, FPI scores) and clinical assessments of the PROMIS-29 survey. Components within the PROMIS-29 survey used for analysis included evaluations of Physical Function, Pain Impact Score, Fatigue, Pain Interference, Depression, Anxiety, Ability to Participate in Social Roles, Global Pain, and Sleep Disturbance. An $\alpha-$ level of 0.05 was established as the level of significance for the study.

\section{Results}

\section{Cohort Characteristics}

A total of 298 unique patients from a single site were included in this retrospective, observational study. The mean age of chronic pain subjects was 60.7 yrs. $(60.68 \pm 13.62)$ with a $57.4 \%$ female population. Raw PROMIS-29 scores were transformed to standardized T-score metrics, with a mean of 50 and standard deviation of 10. PROMIS-29 T-scores for the
Table I PROMIS-29 and T-Score Metrics in Chronic Pain Patients $(n=298)$

\begin{tabular}{|l|c|c|}
\hline \multirow{2}{*}{ PROMIS-29 Domain } & \multicolumn{2}{|c|}{ PROMIS-29 Pain Patients } \\
\cline { 2 - 3 } & Mean \pm SD & Range \\
\hline Global Pain & $7.0 \pm 1.8$ & $1-10$ \\
\hline Pain Impact Score & $36.4 \pm 7.60$ & $15.0-50.0$ \\
\hline & T-Score & \\
\hline Pain Interference & $67.1 \pm 5.79$ & $41.6-75.6$ \\
\hline Ability to Participate in Social Roles & $37.3 \pm 6.39$ & $27.5-53.6$ \\
\hline Sleep Disturbance & $59.9 \pm 7.53$ & $37.6-73.3$ \\
\hline Fatigue & $61.6 \pm 8.39$ & $33.7-75.8$ \\
\hline Depression & $58.2 \pm 9.78$ & $41.0-79.3$ \\
\hline Anxiety & $58.1 \pm 9.22$ & $40.3-81.4$ \\
\hline Physical Function & $34.8 \pm 6.20$ & $22.9-56.9$ \\
\hline
\end{tabular}

Abbreviations: PROMIS-29, Patient-Reported Outcomes Measurement Information System 29-item profile; SD, standard deviation.

7 subdomains along with an 11-point pain intensity measure and pain impact score can be seen in Table 1 .

Consistent with our previously published population profile of chronic pain patients, subjects in this cohort reflected chronic pain profiles of baseline PROMIS-29 subdomain values significantly worse than the general population, which has a mean T-score of $50 .{ }^{17}$ On average, subjects displayed moderate-severe pain (7/10) and moderate-high impact pain (36.4/50). Further, subjects displayed severe pain interference and physical dysfunction, with an average T-score of 67 , which is 1.7 standard deviations worse than that of the US general population. This population also displayed a worsening-shift towards depression, anxiety, fatigue, and sleep disturbance of one standard deviation as compared to the general population. In summary, this represents a population of individuals with severe chronic pain and pain interference that impacts activities of daily living and represents the broader chronic pain population in terms of their multidimensional psychometric profile.

Abnormal biomarker results were detected in a large majority of the chronic pain subjects with $87.2 \%$ of patients exhibiting at least one abnormal biomarker finding. All biomarker results were interpreted using established clinical reference ranges that had been previously determined using pain-free control groups. ${ }^{12,16}$ The most common abnormal biomarker finding among all subjects was elevated quinolinic acid. Quinolinic acid is a neurotoxic NMDA agonist and 
product of the kynurenine pathway of tryptophan catabolism. These results corroborate an earlier study from our group that identified elevated levels of quinolinic acid as the most common abnormal finding in a retrospective analysis of 17,834 chronic pain patients. ${ }^{16}$ Rates of abnormal detection for each component biomarker of the FPI can be seen in Table 2 .

Further supporting the role of an up-regulated kynurenine pathway in chronic pain disorders was the finding of elevated levels of kynurenic acid in 33\% of patients. Elevated pyroglutamate, indicative of glutathione depletion, was detected in $35 \%$ of patients. Glutathione depletion is a common finding in chronic disease states and especially prevalent in the chronic pain population due to the widespread use of analgesic medications containing acetaminophen, which directly depletes glutathione stores. ${ }^{18}$ Elevated levels of xanthurenic acid, a surrogate marker of Vitamin B6 status was detected in $33 \%$ of patients. Increased utilization of pyridoxal-5-phosphate (P5P), the active form of vitamin $\mathrm{B} 6$, is common in inflammatory diseases due to the role that P5P plays as a cofactor in many inflammatory pathways including the kynurenine pathway. ${ }^{19}$

The mean FPI score (0-100 scale) among the chronic pain cohort was $35.9(35.9 \pm 25.2)$ with $17.6 \%$ of patients recording a "Low" FPI score (0-19), 58.1\% of patients recording a "Moderate" FPI score (20-49), 16.6\% of patients recording a "Moderately High" FPI score (50-79), and 7.6\% of patients recording a "High" FPI score (80-100). Table 3 details FPI scores and the average number of abnormal component biomarkers for each of the scoring categories. The number of abnormal biomarker findings per patient ranged from 0 to 7 (out of a total of 11 biomarkers) (Table 4).

\section{Associations of Component Biomarkers with PROMIS-29 Domains}

Correlation analysis was performed using individual PROMIS-29 domains and component biomarker data (ie, individual metabolites of the FPI) for the purpose of identifying associations between individual biomarkers and specific symptomologies. Foundation Pain Index scoring (0100 scale) provides an extremely intuitive means of determining the potential role of biochemistry in a patient's pain. However, to modulate underlying aberrations, it is important to understand the individual biomarkers of the FPI and their mechanistic links to certain symptoms. The serotonin metabolite 5-hydroxyindolacetic acid (5-HIAA), which can indicate abnormal serotonin synthesis and metabolism,

Table 2 Concentrations of Biomarkers and Prevalence of Abnormal Biomarker Findings in Chronic Pain Patients

\begin{tabular}{|l|c|c|c|c|}
\hline \multicolumn{2}{|c|}{} & \multicolumn{3}{c|}{ PROMIS-29 Pain Patients } \\
\hline Biomarker & Reference Range & Mean \pm SD & Range & Prevalence of Abnormal Results \\
\hline Methylmalonic acid & $0-2.3$ & $1.20 \pm 0.70$ & $0-4.3$ & $6.2 \%$ \\
Homocysteine & $0-1.3$ & $0.86 \pm 0.69$ & $0.1-6.7$ & $13 \%$ \\
Xanthurenic acid & $0-0.63$ & $0.58 \pm 0.44$ & $0-4.13$ & $33 \%$ \\
Pyroglutamate & $8-40$ & $38.6 \pm 23.1$ & $0-187$ & $35 \%$ \\
Vanilmandelate & $\geq 0.8$ & $3.43 \pm 1.54$ & $0-9.9$ & $0.7 \%$ \\
5-hydroxyindoleacetic acid & $\geq 1.6$ & $4.78 \pm 3.95$ & $0-34.1$ & $4.2 \%$ \\
Quinolinic acid & $0-6.3$ & $6.12 \pm 2.84$ & $0-23.8$ & $36 \%$ \\
Kynurenic acid & $0-2.0$ & $1.83 \pm 1.05$ & $0-8.4$ & $33 \%$ \\
Hydroxymethylglutarate & $0-5.1$ & $3.39 \pm 1.88$ & $0-17.4$ & $12 \%$ \\
Ethylmalonic acid & $0-6.3$ & $3.75 \pm 2.75$ & $0-22.6$ & $10 \%$ \\
3-hydroxypropyl mercapturic acid & $0-2.4$ & $1.08 \pm 1.73$ & $0-15.3$ & $12 \%$ \\
\hline
\end{tabular}

Abbreviations: PROMIS-29, Patient-Reported Outcomes Measurement Information System 29-item profile; SD, standard deviation.

Table 3 Foundation Pain Index Scoring Characteristics Among PROMIS-29 Patients

\begin{tabular}{|l|c|c|c|c|}
\hline FPI Category & LOW & MODERATE & MOD. HIGH & HIGH \\
\hline FPI Score Range & $0-19$ & $20-49$ & $50-79$ & $80-100$ \\
Average Number of Abnormal Biomarkers & $0.25 \pm 0.44$ & $1.63 \pm 0.61$ & $3.38 \pm 0.49$ & $5.14 \pm 0.83$ \\
Number of Patients in FPI Score Category & 51 & 168 & 48 & 22 \\
Percentage of Patients in Category & $17.6 \%$ & $58.1 \%$ & $16.6 \%$ & $7.6 \%$ \\
\hline
\end{tabular}

Abbreviations: FPI, Foundation Pain Index; PROMIS-29, Patient-Reported Outcomes Measurement Information System 29-item profile; MOD. HIGH, Moderately High. 
Table 4 Prevalence of the Number of Abnormal Biomarker Findings Among Patients

\begin{tabular}{|l|c|c|}
\hline $\begin{array}{l}\text { Number of Abnormal } \\
\text { Biomarkers }\end{array}$ & $\begin{array}{c}\text { Number of } \\
\text { Patients }\end{array}$ & $\begin{array}{c}\text { \% of Patients } \\
\text { (N=289) }\end{array}$ \\
\hline 0 & 38 & $13.1 \%$ \\
1 & 87 & $30.1 \%$ \\
2 & 82 & $28.4 \%$ \\
3 & 42 & $14.5 \%$ \\
4 & 23 & $8.0 \%$ \\
5 & 10 & $3.5 \%$ \\
6 & 6 & $2.1 \%$ \\
7 & 1 & $0.3 \%$ \\
\hline
\end{tabular}

strongly associated with pain interference $(\mathrm{P}=0.046)$, physical function $(\mathrm{P}=0.037)$, and pain impact scores $(\mathrm{P}=$ 0.019). Hydroxymethylglutarate accumulates under conditions of Coenzyme Q10 deficiency and strongly associated with physical function $(\mathrm{P}=0.009)$. Quinolinic acid, a neurotoxic metabolite of the kynurenine pathway (KP) of tryptophan catabolism, exhibited an extremely strong correlation with physical function $(\mathrm{P}<0.0001)$ and less strong associations with pain interference $(\mathrm{P}=0.096)$ and ability to participate in social roles $(\mathrm{P}=0.058)$. Kynurenic acid, another neuroactive metabolite of the KP, strongly associated with pain interference $(\mathrm{P}=0.015)$ and physical function $(\mathrm{P}=0.020)$ and less strongly with pain impact scores $(\mathrm{P}=0.061)$. Levels of homocysteine, a biomarker of $\mathrm{B}$-vitamin status, also associated with pain impact scores $(\mathrm{P}=0.049)$ (Table S1).

Overall, the physical function domain of the PROMIS29 strongly associated with four component biomarkers (5-hydroxyindoleacetic acid, quinolinic acid, kynurenic acid and hydroxymethylglutarate) of the FPI and approached significant associations with xanthurenic acid (biomarker of Vitamin B6 status) and vanilmandelate (metabolite of epinephrine/norepinephrine). Pain interference and the pain impact domains were also associated with numerous component biomarkers of the FPI, further supporting initial clinical validation findings.

\section{Association of FPI Scores with PROMIS-29 Domains}

Criterion analyses validated the presence and significance of any correlations between FPI scores and individual domains of the PROMIS-29. Overall, FPI scores were significantly associated with multiple PROMIS-29 domains including physical function $(\mathrm{P}=0.0023)$, impact score $(\mathrm{P}=0.0033)$, fatigue ( $\mathrm{P}=0.0138)$, pain interference $(\mathrm{P}=0.0223)$, and depression $(\mathrm{P}=0.0473)$ (Table 5). Extremely strong associations between FPI scores and the physical function domain of the PROMIS-29 are consistent with the numerous statistically significant correlations between component biomarkers of the FPI and physical function (Table S1).

\section{Discussion}

These results provide further evidence of clinical validity and confirm the presence of strong associations between FPI scores and validated clinical assessments in chronic pain patients.

This cross-validation of the FPI with PROMIS-29 domains will facilitate further clinical adoption of this innovative laboratory offering as a growing number of pain physicians employ the PROMIS-29 for initial assessment and evaluation of ongoing treatment strategies. Validating biochemical correlates to PROMIS-29 domains not only improves our understanding of pain biochemistry but also provides novel treatment strategies as abnormal biochemical function can be modulated or corrected with safe, non-opioid therapies. ${ }^{20}$ The introduction of targeted, non-opioid therapies capable of alleviating pain and improving PRO measures would represent a significant advancement in the field of pain management.

Furthermore, discovery and validation of biochemical correlates to PRO instruments such as the PROMIS-29 and SF-36 also holds tremendous promise for improving outcomes and patient selection criteria for interventional pain procedures. There is a rapidly growing realization that

Table 5 Association of PROMIS-29 Domains and Foundation Pain Index Scores Among Chronic Pain Patients*

\begin{tabular}{|l|c|c|c|}
\hline X Variable & Y Variable & R (rank) & P-value \\
\hline PROMIS - Physical Function & FPI Score & -0.1758 & 0.0023 \\
Impact Score & FPI Score & 0.2026 & 0.0033 \\
PROMIS - Fatigue & FPI Score & 0.1423 & 0.0138 \\
PROMIS - Pain Interference & FPI Score & 0.1321 & 0.0223 \\
PROMIS - Depression & FPI Score & 0.1148 & 0.0473 \\
PROMIS - Anxiety & FPI Score & 0.09149 & 0.1144 \\
PROMIS - Ability to & FPI Score & -0.07406 & 0.2016 \\
Participate Social & & & \\
PROMIS - Global Pain & FPI Score & 0.04403 & 0.5277 \\
PROMIS - Sleep Disturbance & FPI Score & 0.002828 & 0.9612 \\
\hline
\end{tabular}

Note: *Statistical analysis was performed using Spearman rank's ( $r$ ) coefficient. Abbreviations: PROMIS-29, Patient-Reported Outcomes Measurement Information System 29-item profile; FPI, Foundation Pain Index; R (rank), Spearman correlation coefficient. 
optimal surgical outcomes are heavily dependent on the response rate of patients to a physical and emotional healthy state. ${ }^{21}$ Because of this, the pre-surgical period should be viewed as an effective and opportunistic time to optimize the health status of a patient and maximize the likelihood of a successful outcome. Pre-surgical patient preparation aimed at enhancing surgical recovery, known as "prehabilitation," is a growing field in pain management with tremendous upside for patients, providers, employers, and payors. Most agree that successful prehabilitation requires the use of a multi-modal approach to maximize

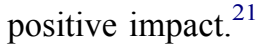

Evaluation of a patient's biochemical status and attempted correction of any deranged biochemical function should be included as part of an innovative approach to optimizing surgical outcomes. Optimizing nutritional status in the perioperative period is currently recommended by the Neurostimulation Appropriateness Consensus Committee (NACC) due to the recognition that malnutrition impairs wound healing and increases susceptibility to infections. ${ }^{22}$ In the context of surgery, malnutrition has been found to be an independent predictor of morbidity and mortality in postoperative patients. ${ }^{23}$ The impact of poor nutritional status on the outcomes of surgery is just one example of how biochemical function can impact patient outcomes. Optimizing biochemical function, which includes but is not limited to nutritional components, has the capacity to improve patient outcomes beyond healing and infection mitigation. In large part, this is due to the potential impact of presurgical biochemical status on the postsurgical inflammatory response. The human response to surgery is intended to be protective in nature and promote successful healing. The cascade of events that follow injury and the stress of surgery is highly complicated and involves an interplay of metabolic, hemodynamic, hormonal, and immunological systems. ${ }^{24}$ An imbalance between the proinflammatory and anti-inflammatory response to surgical intervention may result in an exaggerated or inappropriate response leading to poor outcomes. ${ }^{24}$ While the prognostic value of preoperative nutritional and inflammatory status has been well described in the literature, there remains a limited number of intuitive and cost-effective laboratory tools capable of characterizing the preoperative biochemical status of a patient. Our group will be conducting prospective studies to determine if the Foundation Pain Index can guide preoperative biochemical optimization with the goal of improving patient outcomes across the spectrum of interventional pain procedures. Specifically, our group is interested in determining if pre- and post-operative optimization of biochemical function in the chronic pain population can improve long-term outcomes for surgical interventional procedures such as spinal cord stimulation (SCS).

\section{Conclusion}

Cross-validation of the FPI with the PROMIS-29 in a cohort of 298 chronic pain patients further supports the role of deranged biochemical function in the etiology of chronic pain. Higher FPI scores, indicative of more severely deranged biochemical function, were associated with worsening PROMIS-29 scores across most domains including physical function, pain impact scores, fatigue, pain interference, and depression. Objective identification of underlying biochemical pain determinants or contributing factors will provide practitioners with novel treatment options aimed at correcting underlying biochemical function. Robust, biomarker-based correlates to the complex neurobiological underpinnings of chronic pain will provide novel insight into chronic pain mechanisms and afford non-invasive, longitudinal monitoring. Further research efforts will aim to determine the impact of pre-surgical biochemical correction on surgical outcomes in patients with chronic pain.

\section{Abbreviations}

PRO, patient-reported outcome; NRS, numerical rating scales; VAS, visual analog scales; HHS; Health and Human Services; NIH, National Institutes of Health; IOM, Institute of Medicine; IMMPACT, Initiative on Methods, Measurement, and Pain Assessment in Clinical Trials; FPI, Foundation Pain Index; PROMIS, Patient Reported Outcomes Measurement Information System; SD, standard deviation; PIS, Pain Impact Score; LIS, Laboratory Information System; LC-MS /MS, liquid chromatography tandem mass spectrometry; SCS, spinal cord stimulation; MOD. HIGH, Moderately High; MMA, methylmalonic acid; HCYS, homocysteine; XAN, xanthurenic acid; PGA, pyroglutamic acid; VMA, vanilmandelate; 5-HIAA, 5-hydroxyindoleacetic acid; QA, quinolinic acid; KYNA, kynurenic acid; HMG, hydroxymethylglutarate; EMA, ethylmalonic acid; 3-HPMA, 3-hydroxypropyl mercapturic acid.

\section{Disclosure}

Dr Jason E Pope is a consultant for Ethos Labs, Flowonix, Saluda Medical, PainTEQ, Aurora Spine, SpineThera, Abbott, Medtronic, Vertos, SPARK, SPR Therapeutics, 
WISE, Tersera, Boston Scientific, and AIS, and owns equity in Celeri Health, AGR, and NIS, outside the submitted work. Dr Michael A Fishman is a consultant for Abbott, Aurora Pain Care, Biotronik, Braeburn, Foundation Fusion Solutions, Institute for Musculoskeletal Health, Medtronic, Nevro, Boston Scientific, Cornerloc, Lumbrera, Nalu Medical, Saluda Medical, and StimGenics, and owns equity in Celeri Health, SGX International, and Thermaquil, outside the submitted work. Dr Joshua A Gunn has a patent pending for Methods of Diagnosing and Treating Particular Causal Components of Chronic Pain in a Patient and is Chief Scientific Officer and part-owner of Ethos Research \& Development. Dr Timothy $\mathrm{R}$ Deer is a consultant for Abbott, Flowonix, Mainstay, Ethos Labs, Stimgenics, SI Bone, Nevro, Medtronic, and PainTeq, and owns equity in Vertos Medical, Axonics, SpineThera, Saluda Medical, Nalu, Vertiflex, Cornerloc, SPR Therapeutics, and Boston Scientific, outside the submitted work. In addition, Dr Timothy Deer is the DRG Surgical Leads and has a patent pending to Abbott. The authors report no other conflicts of interest in this work.

\section{References}

1. Gaskin DJ, Richard P. The economic costs of pain in the United States. J Pain. 2012;13(8):715-724. doi:10.1016/j.jpain.2012.03.009

2. Dahlhamer J, Lucas J, Zelaya C, et al. Prevalence of chronic pain and high-impact chronic pain among adults - United States, 2016. MMWR Morb Mortal Wkly Rep. 2018;67(36):1001-1006. doi:10. 15585/mmwr.mm6736a2

3. Dansie EJ, Turk DC. Assessment of patients with chronic pain. $\mathrm{Br}$ J Anaesth. 2013;111(1):19-25. doi:10.1093/bja/aet124

4. Williams DA, Kratz AL. Patient-reported outcomes and fibromyalgia. Rheum Dis Clin North Am. 2016;42(2):317-332. doi:10.1016/j.rdc.20 16.01.009

5. Lawrence RC, Felson DT, Helmick CG, et al. Estimates of the prevalence of arthritis and other rheumatic conditions in the United States. Part II. Arthritis Rheum. 2008;58(1):26-35. doi:10.1002/art. 23176

6. US Dept of Health and Human Services Pain Task Force. Press release. Pain management best practices: updates, gaps, inconsistencies, and recommendations; May 9, 2019.

7. Deyo RA, Dworkin SF, Amtmann D, et al. Report of the NIH Task Force on research standards for chronic low back pain. Phys Ther. 2015;95(2):1-18. doi:10.2522/ptj.2015.95.2.e1

8. Simon LS. Relieving pain in America: a blueprint for transforming prevention, care, education, and research. J Pain Pall Care Pharmacol. 2012;26:197-198.

9. Dworkin RH, Turk DC, Farrar JT, et al. Core outcome measures for chronic pain clinical trials: IMMPACT recommendations. Pain. 2005;113(1-2):9-19. doi:10.1016/j.pain.2004.09.012
10. Dworkin RH, Turk DC, Peirce-Sandner S, et al. Research design considerations for confirmatory chronic pain clinical trials: IMMPACT recommendations. Pain. 2010;149(2):177-193. doi:10.10 16/j.pain.2010.02.018

11. Gewandter JS, Dworkin RH, Turk DC, et al. Research designs for proof-of-concept chronic pain clinical trials: IMMPACT recommendations. Pain. 2014;155(9):1683-1695. doi:10.1016/j.pain. 2014.05.025

12. Amirdelfan K, Pope JE, Gunn J, et al. Clinical validation of a multi-biomarker assay for the evaluation of chronic pain patients in a cross-sectional, observational study. Pain Ther. 2020;9(2):511-529. doi:10.1007/s40122-020-00175-3

13. Cella D, Schalet BD, Kallen M, et al. Press release. Prosetta Stone ${ }^{\circledR}$ analysis report a Rosetta stone for patient reported outcomes: PROMIS pain interference and SF-36/BP; 2013.

14. Choi SW, Podrabsky T, McKinney N, et al. Press release. A Rosetta stone for patient reported outcomes: promis physical function and SF-36 physical function; 2013.

15. Raja SN, Carr DB, Cohen M, et al. The revised International Association for the Study of Pain definition of pain: concepts, challenges, and compromises. Pain. 2020;161(9):1976-1982. doi:10.10 97/j.pain.0000000000001939

16. Gunn J, Hill MM, Cotten BM, Deer TR. An analysis of biomarkers in chronic pain patients. Pain Physician. 2020;23(1):41-49. doi:10. 36076/ppj.2020/23/E41

17. Pope JE, Fishman MA, Chakravarthy K, et al. A retrospective, multicenter, quantitative analysis of patients' baseline pain quality (PROMIS-29) entering into pain and spine practices in the United States (ALIGN). Pain Ther. 2021;10(1):539-550. doi:10.1007/s40 122-021-00238-Z

18. Emmett M. Acetaminophen toxicity and 5-oxoproline (pyroglutamic acid): a tale of two cycles, one an ATP-depleting futile cycle and the other a useful cycle. Clin J Am Soc Nephrol. 2014;9(1):191-200. doi: $10.2215 / \mathrm{CJN} .07730713$

19. Ciorba MA. Kynurenine pathway metabolites: relevant to vitamin B-6 deficiency and beyond. Am J Clin Nutr. 2013;98(4):863-864. doi:10.3945/ajcn.113.072025

20. R Miranda-Massari J, J Gonzalez M, Jimenez FJ, Z Allende-Vigo MZ, Duconge J. Metabolic correction in the management of diabetic peripheral neuropathy: improving clinical results beyond symptom control. Curr Clin Pharmacol. 2011;6(4):260-273. doi:10.2174/ 157488411798375967

21. Scheede-Bergdahl C, Minnella EM, Carli F. Multi-modal prehabilitation: addressing the why, when, what, how, who and where next? Anaesthesia. 2019;74:20-26. doi:10.1111/anae.14505

22. Deer TR, Provenzano DA, Hanes M, et al. The Neurostimulation Appropriateness Consensus Committee (NACC) recommendations for infection prevention and management. Neuromodulation. 2017;20(1):31-50. doi:10.1111/ner.12565

23. Kehlet H. Multimodal approach to control postoperative pathophysiology and rehabilitation. Br J Anaesth. 1997;78(5):606-617. doi:10. 1093/bja/78.5.606

24. Paruk F, Chausse JM. Monitoring the post surgery inflammatory host response. J Emerg Crit Care Med. 2019;3:47. doi:10.21037/jeccm.20 19.08 .06 


\section{Publish your work in this journal}

The Journal of Pain Research is an international, peer reviewed, open access, online journal that welcomes laboratory and clinical findings in the fields of pain research and the prevention and management of pain. Original research, reviews, symposium reports, hypothesis formation and commentaries are all considered for publication. The manuscript management system is completely online and includes a very quick and fair peer-review system, which is all easy to use. Visit http:// www.dovepress.com/testimonials.php to read real quotes from published authors. 